where $B$ is a constant. Although the two methylene couplings in radical I could not be determined exactly, it is possible to conclude that they do not differ for more than 4 gauss. Using $B \cong 50$ gauss and assuming that the angle $\Varangle \mathrm{H}_{(1)} \mathrm{C}_{(6)} \mathrm{H}_{(5)}$ remains approximately the same as in the undamaged molecule $\left(108^{\circ}\right)$, one finds that the angles $\Phi_{1}$ and $\Phi_{2}$ corresponding to the $\mathrm{C}_{(6)}-\mathrm{H}_{(1)}$ and $\mathrm{C}_{(6)}-\mathrm{H}_{(2)}$ bonds, respectively, do not differ by more than $5^{\circ}$, in contrast to the very large difference in the undamaged

16 K. Morokuma and K. Fukui, Bull. Chem. Soc. Japan 36, 534 [1963]. molecule. If one assumes the rocking conformation of the $-\mathrm{CH}_{2}-$ group, $\left|\Phi_{1}-\Phi_{2}\right|$ is even smaller ${ }^{16}$. Equivalence of the two methylene protons proves their symmetric arrangement with respect to the unpaired electron localized on $\mathrm{C}_{(5)}$, hence to the plane defined by $\mathrm{C}_{(5)}, \mathrm{C}_{(6)}$ and $\mathrm{C}_{(7)}$. Since $\mathrm{C}_{(5)}-\mathrm{C}_{(7)}$ bond lies in the ring plane one safely concludes that in the 5 -thymyl radical the whole pyrimidine ring is approximately planar.

The help of Dr. B. KoJić-Prodić in the X-ray crystal analysis is gratefully acknowledged. This investigation was supported by the Yugoslav Federal Research Fund and by the Research Fund of Croatia.

\title{
Optically Thin Hydrogen Plasma with Self-Consistent Electron Velocity Distribution
}

\author{
J. Oxenius \\ Association Euratom-CEA, Département de Physique du Plasma et de la Fusion Contrôlée, \\ Fontenay-aux-Roses, France \\ (Z. Naturforsch. 25 a, 1302-1310 [1970] ; received 5 February 1970)
}

\begin{abstract}
Occupation numbers of the atomic levels and degree of ionization of a stationary optically thin hydrogen plasma are calculated for temperatures in the range $8000^{\circ} \mathrm{K} \lesssim T \lesssim 16000^{\circ} \mathrm{K}$ and for electron densities in the range $10^{9} \mathrm{~cm}^{-3} \lesssim n_{\mathrm{e}} \lesssim 10^{17} \mathrm{~cm}^{-3}$. The electron velocity distribution is not supposed to be Maxwellian, rather, it is obtained in a self-consistent way from the corresponding kinetic equation which is solved simultaneously with the equations that describe the statistical steady-state of the atomic levels. Besides spontaneous radiative bound-bound transitions and radiative recombinations, inelastic electron-atom collisions as well as elastic electronelectron and electron-atom collisions are taken into account. Hydrogen atoms are approximated by model atoms having four bound levels and a continuum. The numerical results show that for temperatures $T \lesssim 10000^{\circ} \mathrm{K}$ the tail of the electron distribution function differs markedly from that of a Maxwell distribution, except in the case of high electron densities. Likewise, the selfconsistently calculated values of the atomic occupation numbers and of the degree of ionization there deviate strongly, up to several orders of magnitude, from those obtained by assuming a Maxwellian velocity distribution of the electrons.
\end{abstract}

\section{Introduction}

The principle of detailed balancing ensures that in the case of thermal equilibrium the loss of electrons out of the tail of the distribution function due to excitation or ionization collisions with atoms is exactly compensated by the production of such electrons due to the corresponding deexcitation or three-body recombination collisions. If the upper level of any atomic transition is underpopulated relative to the Boltzmann or Saha value ("departure from thermal equilibrium"), this balancing is perturbed, resulting in a net flux of electrons from the high energy tail into the low energy body of the distribution function. In a stationary state, this net

Reprint requests to Dr. J. Oxenius, Association Euratom-CEA, Centre d'Etudes Nucléaires, Boîte Postale No 6, F-92 Fontenay-aux-Roses, France. flux is then counterbalanced by elastic electron collisions which tend to fill up the tail of the distribution function to its Maxwellian values. Departures of the atomic occupation numbers from their thermal values thus result in departures of the electron velocity distribution from a Maxwell distribution.

This shows that in determining the spectroscopic state of a plasma a self-consistent procedure has to be applied. For the atomic occupation numbers depend on the collision rates and hence on the electron velocity distribution, which, in turn, depends on the occupation numbers through the mechanism described above. Only in the case of prevailing elastic collisions is the electron distribution function independent of the interaction with the atoms; it is then approximately Maxwellian 
and enters into the calculation of the occupation numbers only through the given parameter "electron temperature."

First investigations of this self-consistency problem have been undertaken by Biberman, VoroBev, and Yakubov ${ }^{1}$, and by Peyraud ${ }^{2}$. BiberMAN et al. try to develop a procedure for the most general optically thin case, based on the idea of diffusion in discrete energy space, and compare their theory with experimental data of a positive argon column. Peyraud investigates the specific case of an optically thick hydrogen plasma by means of model atoms with three discrete levels and a continuum; unfortunately, the cursory treatment of radiative transfer by means of an only estimated "coefficient of imprisonment" introduces once more an inconsistency into the calculations, this time via the radiation field, and may thus lead to considerable numerical errors.

The present paper treats optically thin hydrogen plasmas in a self-consistent way by solving the kinetic equation for the electron velocity distribution simultaneously with the balance equations for the atomic levels. The procedure is straightforward, largely based on results of an investigation of the effect of single bound- bound or free-bound transitions ("two-level atcms") on the electron distribution $^{3}$. In view of numerical calculations that Draw IN ${ }^{4}$ has carried out for model $\mathrm{H}$-atoms having different numbers of bound levels, it was judged sufficient to take only four bound levels and the continuum into consideration. The results thus obtained are indeed in fair agreement with values published by Bates, Kingston, and McWhirter ${ }^{5}$ and by DrawiN ${ }^{4}$, provided, of course, that for the electrons a Maxwell distribution is assumed.

The main result of the present paper will be that for temperatures below $10000{ }^{\circ} \mathrm{K}$ the assumption of a Maxwellian tail of the electron distribution function in optically thin hydrogen plasmas is unjustified for most electron densities of practical interest, and that accordingly calculations of atomic occupation numbers and of the degree of ionization based on this assumption lead to results that may be wrong by orders of magnitude.

1 L. M. Biberman, V. S. Vorobev, and I. T. Yakubov, High Temperature 6, 359 [1968].

2 N. Peyraud, Phys. Letters 27 A, 410 [1968]; Thèse, Faculté des Sciences de Paris, Paris 1969.

\section{System}

The system considered is a stationary, homogeneous, and isotropic plasma without external fields, composed of hydrogen atoms, electrons, and protons, the proton density being equal to the electron density. Hydrogen atoms are approximated by model atoms with four bound levels and a continuum, and equipartition over the degenerate states of a level is assumed. All bound-bound and freebound transitions are supposed to be optically thin. The physical processes taken into account are: spontaneous radiative bound-bound transitions; spontaneous radiative recombinations; inelastic electron-atom collisions for excitation, deexcitation, ionization, and three-body recombination; elastic electron-electron collisions; elastic collisions of electrons with $\mathrm{H}$-atoms. Inelastic atom-atom collisions are neglected. The $\mathrm{H}$-atoms are supposed to have a Maxwellian velocity distribution of temperature $T$, and the electrons to have a Maxwellian velocity distribution of the same temperature $T$ for energies that are smaller than the lowest resonance energy of a hydrogen atom.

\section{Basic Equations}

We first define dimensionless quantities to be used in the following.

Instead of energy $E$, temperature $T$, electron density $n_{\mathrm{e}}$, and proton density $n_{+}$, we introduce

$$
\begin{gathered}
\varepsilon=E / I_{\mathrm{H}}, \\
\vartheta=I_{\mathrm{H}} / k T, \\
v_{\mathrm{e}}=n_{\mathrm{e}} a_{0}{ }^{3}, \quad v_{+}=n_{+} a_{0}^{3}
\end{gathered}
$$

( $I_{\mathrm{H}}=e_{0}^{2} / 2 a_{0}=$ Rydberg unit of energy,

$a_{0}=\hbar^{2} / m e_{0}^{2}=$ Bohr radius,

$e_{0}=$ elementary charge, $m=$ electron mass).

The electrical neutrality of the plasma requires

$$
n_{+}=n_{\mathrm{e}}, \quad \boldsymbol{v}_{+}=\boldsymbol{v}_{\mathrm{e}} .
$$

Let $E_{i}$ be the ionization energy of the level with principal quantum number $i$ of an $\mathrm{H}$-atom, and $E_{i j}=E_{i}-E_{j}(i<j)$ the energy difference of levels $i$ and $j$; the corresponding dimensionless quantities are then $\varepsilon_{i}=1 / i^{2}$ and $\varepsilon_{i j}=\varepsilon_{i}-\varepsilon_{j}$.

3 J. Oxenius, Z. Naturforsch 25a, 101 [1970].

4 H. W. Drawin, Ann. Phys. Leipzig 14, 262 [1964].

5 D. R. Bates, A. E. Kingston and R. W. P. McWhirter, Proc. Roy. Soc. London A 267, 297 [1962]. 
As in Ref. ${ }^{3}$, we define numbers $\alpha_{i j}$ and $\beta_{i}$ through

$$
\begin{gathered}
n_{j} / n_{i}=\alpha_{i j}\left(j^{2} / i^{2}\right) \exp \left\{-E_{i j} / k T\right\}, \\
n_{\mathrm{e}} n_{+} / n_{i}=\beta_{i} \frac{1}{i^{2}}\left(\frac{m k T}{2 \pi \hbar^{2}}\right)^{3 / 2} \exp \left\{-E_{i} / k T\right\}
\end{gathered}
$$

$\left(n_{i}, n_{j}=\right.$ atom densities). They can be written in terms of the more commonly used $b$-values as

$$
\alpha_{i j}=b_{j} / b_{i}, \quad \beta_{i}=1 / b_{i},
$$

$b_{i}$ being defined by

$$
b_{i}=n_{i} / n_{i}^{\text {Saha }}
$$

where $n_{i}^{\text {Saha }}$ denotes the value of $n_{i}$ that follows from Saha's equation using the densities $n_{\mathrm{e}}$ and $n_{+}$ actually present.

The degree of ionization will be characterized by the quantity

$$
\chi=n_{\mathrm{H}} / n_{+}, \quad n_{\mathrm{H}}=\sum_{i} n_{i} .
$$

Finally, instead of the normalized electron distribution function $f(E)$ we introduce, as in Ref. ${ }^{3}$, the reduced distribution function $\gamma(\varepsilon)$ through

$$
f(E)=\gamma(\varepsilon) \frac{2 E^{1 / 2}}{\pi^{1 / 2}(k T)^{3 / 2}} \exp \{-E / k T\} .
$$

We will suppose that the electron distribution function can be considered Maxwellian for all energies $E$ that are smaller than the lowest resonance energy $E_{12}$ of a hydrogen atom, i.e. that $\gamma(\varepsilon)=1$ for $0 \leqq \varepsilon \leqq \varepsilon_{12}$. Thus only the effect of the resonance transitions on the electron distribution function will be taken into account. This seems to be justified as a first approximation because the densities of excited atoms are very low.

\subsection{Balance Equations of the Atomic Levels}

We write (cf. Ref. $\left.{ }^{3}\right)$ the cross section for collisional excitation of level $j$ from level $i(i<j)$

$$
Q_{i j}(E)=\pi a_{0}^{2}\left(\frac{2 I_{\mathrm{H}}}{E_{i j}}\right)^{2} f_{i j} \varphi_{i j}\left(\frac{E}{E_{i j}}\right)
$$

$\left(f_{i j}=\right.$ absorption oscillator strength $)$, and the cross section for collisional ionization from level $i$

$$
Q_{i}(E)=\pi a_{0}^{2}\left(\frac{2 I_{\mathrm{H}}}{E_{i}}\right)^{2} \varphi_{i}\left(\frac{E}{E_{i}}\right)
$$

As functions $\varphi$ we take

$$
\begin{aligned}
\varphi_{12}(u) & =(0,58 / u) \ln (1,3 u), \\
\varphi_{1}(u) & =0,6 \varphi_{0}(u),
\end{aligned}
$$

and for all other $\varphi_{i j}$ and $\varphi_{i}$

$$
\varphi_{0}(u)=\frac{u-1}{u^{2}} \ln (1,25 u) .
$$

The function $\varphi_{12}$, which is finite at threshold $u=1$, has been determined by approximating the cross sections $Q(1 \mathrm{~s} \rightarrow 2 \mathrm{p})$ and $Q(1 \mathrm{~s} \rightarrow 2 \mathrm{~s})$ calculated by BURKE and collaborators using the close coupling approximation ${ }^{6}$.

The rates of the different collisional and radiative processes can then be written in the following way:

a) Collisional excitation $i \rightarrow j$

$$
n_{i} n_{\mathrm{e}} C_{i j}=\lambda b_{i} \xi_{i j}
$$

b) Collisional deexcitation $j \rightarrow i$

$$
n_{j} n_{\mathrm{e}} D_{j i}=\lambda b_{j} \xi_{i j}^{(\mathrm{o})}
$$

c) Collisional ionization from level $i$

$$
n_{i} n_{\mathrm{e}} S_{i}=\lambda b_{i} \xi_{i}
$$

d) Three-body recombination into level $i$

$$
n_{+} n_{\mathrm{e}}^{2} T_{i}=\lambda \xi_{i}^{(\mathrm{o})}
$$

e) Spontaneous radiative transition $j \rightarrow i$

$$
n_{j} A_{j i}=\lambda b_{j} \eta_{i j}
$$

f) Spontaneous radiative recombination into level $i$

$$
n_{+} n_{\mathrm{e}} R_{i}=\lambda \eta_{i}
$$

The $b_{i}$ are defined by Eq. (8), $\lambda$ stands for

$$
\lambda=2^{6} \pi^{2} \alpha c a_{0}^{5} \vartheta^{3} n_{e}^{2} n_{+}
$$

$\left(\alpha=e_{0}{ }^{2} / \hbar c=\right.$ fine structure constant), and the $\xi$ 's and $\eta$ 's are given by

$\xi_{i j}=i^{2} e^{\vartheta \varepsilon_{i}}\left(f_{i j} / \varepsilon_{i j}\right) \int_{\varepsilon_{i j}}^{\infty} \gamma(\varepsilon)\left(\varepsilon / \varepsilon_{i j}\right) \varphi_{i j}\left(\varepsilon / \varepsilon_{i j}\right) e^{-\vartheta \varepsilon} \mathrm{d} \varepsilon$,

$\left.\xi_{i j}^{(o)}=i^{2} e^{\vartheta \varepsilon_{i}}\left(f_{i j} / \varepsilon_{i j}\right) \int_{\varepsilon_{i j}}^{\infty}\left(\varepsilon / \varepsilon_{i j}\right) \varphi_{i j}\right)\left(\varepsilon / \varepsilon_{i j}\right) e^{-\vartheta \varepsilon} \mathrm{d} \varepsilon$,

$\xi_{i}=i^{2} e^{\vartheta \varepsilon_{i}}\left(\mathbf{1} / \varepsilon_{i}\right) \int_{\varepsilon_{i}}^{\infty} \gamma(\varepsilon)\left(\varepsilon / \varepsilon_{i}\right) \varphi_{i}\left(\varepsilon / \varepsilon_{i}\right) e^{-\vartheta \varepsilon} \mathrm{d} \varepsilon$,

$\xi_{i}^{(0)}=i^{2} e^{\vartheta \varepsilon_{i}}\left(1 / \varepsilon_{i}\right) \int_{\varepsilon_{i}}^{\infty}\left(\varepsilon / \varepsilon_{i}\right) \varphi_{i}\left(\varepsilon / \varepsilon_{i}\right) e^{-\vartheta \varepsilon} \mathrm{d} \varepsilon$,

$\eta_{i j}=\left(\alpha^{3} / 16 \pi^{1 / 2}\right)\left(\nu_{\mathrm{e}} \vartheta^{3 / 2}\right)^{-1} i^{2} f_{i j} \varepsilon_{i j}{ }^{2} e^{\vartheta \varepsilon_{j}}$,

$\eta_{i}=\alpha^{3}(3 \pi)^{-3 / 2}\left(\nu_{\mathrm{e}} \vartheta^{3 / 2}\right)^{-1}\left(\Gamma_{i} / i^{3}\right) e^{\vartheta \varepsilon_{i}} E_{1}\left(\vartheta \varepsilon_{i}\right)$

( $\Gamma_{i}=$ Gaunt factor; we take $\Gamma_{1}=0,8$ and $\Gamma_{i}=1$ otherwise. $E_{1}(x)=\int_{x}^{\infty}\left(e_{m}^{-t} / t\right) \mathrm{d} t=$ exponential in
tegral).

The rate coefficients $D_{j i}, T_{i}$, and $R_{i}$ have been calculated using a Maxwellian electron distribution as is certainly justified since primarily electrons of 
low energy are involved in these downward processes. In determining $R_{i}$, use has been made of Kramers' semi-classical cross section for photoionization.

The balance equations that describe the statistical steady-state of the atomic levels then take the form

$$
\sum_{k=1}^{4} B_{i k} b_{k}=B_{i} \quad(i=1,2,3,4)
$$

with the coefficients

$$
\begin{aligned}
& B_{i i}=\sum_{k<i}\left(\xi_{k i}^{(o)}+\eta_{k i}\right)+\sum_{k>i} \xi_{i k}+\xi_{i}, \\
& B_{i j}=-\left(\xi_{i j}^{(o)}+\eta_{i j}\right) \quad(i<j), \\
& B_{j i}=-\xi_{i j} \quad(i<j), \\
& B_{i}=\xi_{i}^{(0)}+\eta_{i} \text {. }
\end{aligned}
$$

\subsection{Kinetic Equation of the Electrons}

The assumptions of stationarity, homogeneity, and absence of external fields reduce the kinetic equation for the electron distribution function to the requirement that the collision terms due to elastic and inelastic collisions cancel each other:

$$
(\partial f / \partial t)_{\mathrm{el}}+(\partial f / \partial t)_{\mathrm{inel}}=\mathbf{0} .
$$

3.2.1 Elastic collision term. The FokkerPlanck collision term due to elastic electron-electron collisions, valid for the high energy tail of the distribution function, is given by ${ }^{7}$

$$
\left(\frac{\partial f}{\partial t}\right)_{\mathrm{el}}^{\mathrm{ee}}=\frac{m^{3 / 2} n_{\mathrm{e}} \Gamma}{2^{1 / 2}} \frac{\mathrm{d}}{\mathrm{d} E}\left[\frac{f(E)}{E^{1 / 2}}+k T \frac{\mathrm{d}}{\mathrm{d} E}\left(\frac{f(E)}{E^{1 / 2}}\right)\right]
$$

where

$$
\Gamma=\frac{4 \pi e_{0}^{4}}{m^{2}} \ln \Lambda, \quad \Lambda=\frac{3(k T)^{3 / 2}}{2 \pi^{1 / 2} e_{0}^{3} n_{e}^{1 / 2}} .
$$

Collision term (27) implies in agreement with our assumptions that $f(E)$ differs from a Maxwell dis- tribution only for energies $E \gg k T$ so that it still makes sense to speak of an electron temperature $T$.

The corresponding electron-proton collision term is smaller than term (27) by the factor $n_{+} m /$ $n_{e} \boldsymbol{M}_{p}=m / \boldsymbol{M}_{p}\left(\boldsymbol{M}_{p}=\right.$ proton mass $)$, and is hence negligible.

The collision term due to elastic collisions of electrons with $\mathrm{H}$-atoms is given by ${ }^{8,9}$

$$
\begin{aligned}
\left(\frac{\partial f}{\partial t}\right)_{\mathrm{el}}^{\mathrm{eH}}= & \frac{2^{3 / 2} m^{1 / 2} n_{1}}{M} \frac{\mathrm{d}}{\mathrm{d} E}\left\{E ^ { 2 } Q _ { \mathrm { eH } } ( E ) \left[\frac{f(E)}{E^{1 / 2}}\right.\right. \\
& \left.\left.+k T \frac{\mathrm{d}}{\mathrm{d} E}\left(\frac{f(E)}{E^{1 / 2}}\right)\right]\right\}
\end{aligned}
$$

( $M=\mathrm{H}$-atom mass). As total cross section $Q_{\mathrm{eH}}$ for elastic scattering of electrons by $\mathrm{H}$-atoms we take ${ }^{10}$

$$
Q_{\mathrm{eH}}(E)=\pi a_{0}^{2} 6,5\left(I_{\mathrm{H}} / E\right)^{1 / 2} .
$$

Collision term (29) describes the effect of elastic collisions of (light) electrons with (heavy) $\mathrm{H}$-atoms $(m \ll M)$ that are supposed to have a Maxwellian velocity distribution of temperature $T$; the electron energy $E$ has to satisfy $E \gg(m / M) k T$. Only collisions with $\mathrm{H}$-atoms in the ground state 1 have been taken into account, and an isotropic differential cross section has been assumed.

The total elastic collision term of the electron distribution function is hence

$$
(\partial f / \partial t)_{\mathrm{el}}=(\partial f / \partial t)_{\mathrm{el}}^{\mathrm{ee}}+(\partial f / \partial t)_{\mathrm{el}}^{\mathrm{eH}} .
$$

3.2.2. Inelastic collision term. According to our approximation, only the effect of the resonance transitions on the electron distribution funcion will be taken into account.

The collision term due to excitation and deexcitation collisions for the transition between the ground level 1 and the excited level $i$ is for energies $E \geqq$ excitation energy $E_{1 i}$ given by ${ }^{3}$

$$
\left(\frac{\partial f}{\partial t}\right)_{\text {inel }}^{1 i}=-n_{1}\left(\frac{2 E}{m}\right)^{1 / 2} Q_{1 i}(E) f(E)+n_{i} \frac{1}{i^{2}}\left(\frac{2}{m}\right)^{1 / 2}\left(\frac{E^{2}}{E-E_{1 i}}\right)^{1 / 2} Q_{1 i}(E) f\left(E-E_{1 i}\right),
$$

the first term describing the effect of excitation collisions and the second that of deexcitation collisions. The cross section for collisional deexcitation

6 B. L. Moiseiwitsch and S. J. Smith, Rev. Mod. Phys. 40, 238 [1968].

7 M. N. Rosenbluth, W. M. MacDonald, and D.L.Judd, Phys. Rev. 107, 1 [1957].

8 S. Chapman and T. G. Cowling, The Mathematical Theory of Non-Uniform Gases, 2nd ed.: Cambridge University Press, London 1952. has been expressed in Eq. (32) through the cross section for collisional excitation $Q_{1 i}$ via the principle of detailed balancing ${ }^{3}$.

9 E. Moreau and J. Salmon, J. Phys. Radium 21, 217 [1960].

10 B. L. Moiserwitsch, Elastic Scattering of Electrons, in: Atomic and Molecular Processes (ed. D. R. Bates); Academic Press, New York and London 1962. 
The corresponding term due to ionization and three-body recombination collisions is for energies $E \geqq$ ionization energy $E_{1}$ given by ${ }^{3}$

$\left(\frac{\partial f}{\partial t}\right)_{\text {inel }}^{1+}=-n_{1}\left(\frac{2 E}{m}\right)^{1 / 2} Q_{1}(E) f(E)+n_{+} n_{\mathrm{e}} \frac{2 \pi^{2} \hbar^{3}}{m^{2}} \iint\left(\frac{E^{2}}{E^{\prime} E^{\prime \prime}}\right)^{1 / 2} R_{1+}\left(E ; E^{\prime}, E^{\prime \prime}\right) f\left(E^{\prime}\right) f\left(E^{\prime \prime}\right) \mathrm{d} E^{\prime} \mathrm{d} E^{\prime \prime}$,

the first term describing the effect of ionization collisions and the second that of three-body recombination collisions. The transition function $R_{1+}\left(E ; E^{\prime}, E^{\prime \prime}\right)$, which appears in Eq. (33) because the corresponding transition function for three-body recombinations has been expressed through it via the principle of detailed balancing ${ }^{3}$, is proportional to the probability density that the ionization collision of an electron of energy $E$ produces two outgoing electrons with energies $E^{\prime}$ and $E^{\prime \prime}$, respectively; it is related to the cross section for collisional ioni- zation $Q_{1}$ by ${ }^{3}$

$$
Q_{1}(E)=\iint R_{1+}\left(E ; E^{\prime}, E^{\prime \prime}\right) \mathrm{d} E^{\prime} \mathrm{d} E^{\prime \prime} .
$$

The total inelastic collision term of the electron distribution function is hence

$$
(\partial f / \partial t)_{\text {inel }}=\sum_{i=2}^{4}(\partial f / \partial t)_{\text {inel }}^{1 i}+(\partial f / \partial t)_{\text {inel }}^{1+} .
$$

3.2.3. Kinetic equation. Gathering all relevant relations, the kinetic Eq. (26) for the electron distribution function can be written (cf. also Ref. ${ }^{3}$ )

$$
\begin{aligned}
\{1+ & \left.\frac{6,5 m}{M} \frac{n_{1} / n_{\mathrm{e}}}{4 \ln \Lambda} \varepsilon^{3 / 2}\right\} \gamma^{\prime \prime}(\varepsilon)-\vartheta\left\{1+\frac{6,5 m}{M} \frac{n_{1} / n_{\mathrm{e}}}{4 \ln \Lambda} \varepsilon^{3 / 2}\left(1-\frac{3}{2 \vartheta \varepsilon}\right)\right\} \gamma^{\prime}(\varepsilon) \\
& -\frac{\left(n_{1} / n_{\mathrm{e}}\right) \vartheta}{2 \ln \Lambda}\left\{\sum_{i=2}^{4} \frac{f_{1 i}}{\varepsilon_{1 i}} \frac{\varepsilon}{\varepsilon_{1 i}} \varphi_{1 i}\left(\frac{\varepsilon}{\varepsilon_{1 i}}\right)\left[\gamma(\varepsilon)-\alpha_{1 i} \gamma\left(\varepsilon-\varepsilon_{1 i}\right)\right]\right. \\
& \left.+\frac{1}{\varepsilon_{1}} \frac{\varepsilon}{\varepsilon_{1}} \varphi_{1}\left(\frac{\varepsilon}{\varepsilon_{1}}\right)\left[\gamma(\varepsilon)-\frac{\beta_{1}}{q_{1}(\varepsilon)} \iint r_{1+}\left(\varepsilon ; \varepsilon^{\prime}, \varepsilon^{\prime \prime}\right) \gamma\left(\varepsilon^{\prime}\right) \gamma\left(\varepsilon^{\prime \prime}\right) \mathrm{d} \varepsilon^{\prime} \mathrm{d} \varepsilon^{\prime \prime}\right]\right\}=0
\end{aligned}
$$

where the dimensionless transition function $r_{1+}$ and the dimensionless cross section $q_{1}$ are defined by

$$
\begin{aligned}
Q_{1}(E) & =\pi a_{0}^{2} q_{1}(\varepsilon), \\
R_{1+}\left(E ; E^{\prime}, E^{\prime \prime}\right) & =\left(\pi a_{0}{ }^{2} / I_{\mathrm{H}^{2}}\right) r_{1+}\left(\varepsilon ; \varepsilon^{\prime}, \varepsilon^{\prime \prime}\right), \\
q_{1}(\varepsilon) & =\iint r_{1+}\left(\varepsilon ; \varepsilon^{\prime}, \varepsilon^{\prime \prime}\right) \mathrm{d} \varepsilon^{\prime} \mathrm{d} \varepsilon^{\prime \prime} ;
\end{aligned}
$$

furthermore, it is understood that the functions $\varphi$, which contain the energy dependence of the collision cross sections, vanish for energies smaller than the respective threshold energies.

Eq. (36) is valid for $\varepsilon \geqq \varepsilon_{12}$. It has to be supplemented by the equation

$$
\gamma(\varepsilon)=1 \quad \text { if } \quad \varepsilon \leqq \varepsilon_{12}
$$

which expresses the fact that the electron distribution is Maxwellian for energies below the lowest resonance energy $E_{12}$ of a hydrogen atom. In addition, $\gamma(\varepsilon)$ has to satisfy the boundary condition

$$
\gamma(\infty) \text { finite. }
$$

The origin of the different terms of Eq. (36) is as follows: The first two terms, proportional to $\gamma^{\prime \prime}$ and $\gamma^{\prime}$, respectively, are due to elastic collisions, the term " 1 " being due to electron-electron collisions and the term " $(6,5 \mathrm{~m} / \mathrm{M}) \ldots$.. due to electron- atom collisions; the third term, which contains only $\gamma$ itself, is due to inelastic collisions, the term " $\sum$..." being due to bound-bound transitions and the remaining term due to free-bound transitions.

\section{Electron Distribution Function}

We now proceed to find an approximate solution to the kinetic Eq. (36).

Since we are interested in temperatures $T \lesssim 16000{ }^{\circ} \mathrm{K}(\vartheta \gtrsim 10)$, only a rather small $\varepsilon$-interval needs to be considered. Taking $\vartheta=10$ and assuming a Maxwellian electron distribution, one readily estimates that about $90 \%$ of the ionization rate are due to electrons with energies in the range $1 \leqq \varepsilon \leqq 1,4$; the corresponding energy range is still smaller for larger values of $\vartheta$ and/or for nonMaxwellian distributions. This means that a solution of Eq. (36) for $0,75 \leqq \varepsilon \lesssim 1,4$ is all we need in order to calculate the collisional excitation and ionization rates.

If $0,75 \leqq \varepsilon \leqq 1,4$, one has $\gamma\left(\varepsilon-\varepsilon_{1 i}\right)=1$ for all $i$ because of Eq. (38), and in the last term of Eq. (36) the double integral and $q_{1}(\varepsilon)$ cancel out because of Eqs. (38) and (37c).

Since $\vartheta \gg 1$ and $\varepsilon \simeq 1$, the term $3 / 2 \vartheta \varepsilon$ is negligible compared to 1 , leading to identical bracket 
expressions in the first two terms of Eq. (36). Replacing there $\varepsilon^{3 / 2}$ by a constant $\varepsilon_{0}{ }^{3 / 2}$ with $0,75<\varepsilon_{0}<1,4$, one introduces an error which will be of the order of 2 in the case of predominant elastic electron-atom collisions $\left(n_{1} / n_{\mathrm{e}} \ll 10^{4}\right)$ and which is negligible in the opposite limiting case of predominant elastic electron-electron collisions $\left(n_{1} / n_{\mathrm{e}} \ll 10^{4}\right)$.

Thus the following approximate differential equation holds :

$\gamma^{\prime \prime}-\vartheta \gamma^{\prime}-\frac{\left(n_{1} / n_{\mathrm{e}}\right) \vartheta}{2 \omega \ln \Lambda}\left\{\sum_{i=2}^{4} \frac{f_{1 i}}{\varepsilon_{1 i}} \frac{\varepsilon}{\varepsilon_{1 i}} \varphi_{1 i}\left(\frac{\varepsilon}{\varepsilon_{1 i}}\right)\left[\gamma-\alpha_{1 i}\right]\right.$ $\left.+\frac{1}{\varepsilon_{1}} \frac{\varepsilon}{\varepsilon_{1}} \varphi_{1}\left(\frac{\varepsilon}{\varepsilon_{1}}\right)\left[\gamma-\beta_{1}\right]\right\}=0$

where

$$
\omega=1+\frac{6,5 m}{M} \frac{n_{1} / n_{\mathrm{e}}}{4 \ln \Lambda} \varepsilon_{0}^{3 / 2} .
$$

We now replace the numbers $\alpha_{1 i}$ and $\beta_{1}$ by the largest one among them, i.e. by $\alpha_{12}$. This means that we approximate the true distribution function by an upper limit of it since we overestimate the collision rates of the downward transitions. Writing $\alpha_{12}=b_{2} / b_{1}$ [cf. Eq. (7)], we have

$$
\gamma(\varepsilon)=\left(1-\frac{b_{2}}{b_{1}}\right) \gamma_{0}(\varepsilon)+\frac{b_{2}}{b_{1}}
$$

where $\gamma_{0}(\varepsilon)$ obeys for $\varepsilon \geqq \varepsilon_{12}$ the homogenous differential equation

$$
\begin{array}{r}
\gamma_{0}^{\prime \prime}-\vartheta \gamma_{0}^{\prime}-\frac{\left(n_{1} / n_{\mathrm{e}}\right) \vartheta}{2 \omega \ln \Lambda}\left\{\sum_{i=2}^{4} \frac{f_{1 i}}{\varepsilon_{1 i}} \frac{\varepsilon}{\varepsilon_{1 i}} \varphi_{1 i}\left(\frac{\varepsilon}{\varepsilon_{1 i}}\right) \quad\right. \\
\left.+\frac{1}{\varepsilon_{1}} \frac{\varepsilon}{\varepsilon_{1}} \varphi_{1}\left(\frac{\varepsilon}{\varepsilon_{1}}\right)\right\} \gamma_{0}=0
\end{array}
$$

with the boundary condition

in addition,

$$
\gamma_{0}(\infty) \text { finite; }
$$

$$
\gamma_{0}(\varepsilon)=1 \text { if } \varepsilon \leqq \varepsilon_{12} .
$$

As a last step, we approximate the functions $u \varphi(u)$ by linear ones ${ }^{3}$ :

$$
u \varphi_{\mathrm{s}}(u) \simeq k_{\mathrm{s}} u-l_{\mathrm{s}} \quad(u \geqq 1) ;
$$

we take $k_{12}=0,46 ; l_{12}=0,31 ; k_{13}=l_{13}=k_{14}=$ $l_{14}=0,45 ; k_{1}=l_{1}=0,27$.

The functiôn $\gamma_{0}(\varepsilon)$ can then be written down explicitly. One gets

$$
\begin{aligned}
& \left(\mathrm{I}: 0 \leqq \varepsilon \leqq \varepsilon_{12}\right) \gamma_{0}^{(\mathrm{I})}(\varepsilon)=1, \\
& \left(\mathrm{II}: \varepsilon_{12} \leqq \varepsilon \leqq \varepsilon_{13}\right) \\
& \gamma_{0}^{(\mathrm{II})}(\varepsilon)=c_{\mathrm{II}} e^{\vartheta \varepsilon / 2} \operatorname{Ai}\left[\Psi_{\mathrm{II}}(\varepsilon)\right],
\end{aligned}
$$

$$
\begin{aligned}
& \left(\mathrm{III}: \varepsilon_{13} \leqq \varepsilon \leqq \varepsilon_{14}\right) \\
& \gamma_{0}^{(\mathrm{III})}(\varepsilon)=c_{\mathrm{III}} e^{\vartheta \varepsilon / 2} \operatorname{Ai}\left[\Psi_{\mathrm{III}}(\varepsilon)\right], \\
& \left(\mathrm{IV}: \varepsilon_{14} \leqq \varepsilon \leqq \varepsilon_{1}\right) \\
& \gamma_{0}^{(\mathrm{IV})}(\varepsilon)=c_{\mathrm{IV}} e^{\vartheta \varepsilon / 2} \operatorname{Ai}\left[\Psi_{\mathrm{IV}}(\varepsilon)\right] \\
& \left(\mathrm{V}: \varepsilon_{1} \leqq \varepsilon<\infty\right) \\
& \gamma_{0}^{(\mathrm{V})}(\varepsilon)=c_{\mathrm{V}} e^{\vartheta \varepsilon / 2} \operatorname{Ai}\left[\Psi_{\mathrm{V}}(\varepsilon)\right],
\end{aligned}
$$

where $\operatorname{Ai}(x)$ is the tabulated Airy function ${ }^{11}$. The constants $c_{\mathrm{II}}, c_{\mathrm{III}}, c_{\mathrm{IV}}, c_{\mathrm{V}}$, which we do not write down explicitly, are chosen such that $\gamma_{0}$ is continuous, and the functions $\Psi$ are given by

$\Psi_{\mathrm{N}}(\varepsilon)=\vartheta\left(\frac{\varrho \sigma_{\mathrm{N}}}{1+\mu \vartheta^{2} \varrho}\right)^{1 / 3}\left(\varepsilon-\frac{\tau_{\mathrm{N}}}{\sigma_{\mathrm{N}}}+\frac{1+\mu \vartheta^{2} \varrho}{4 \vartheta \varrho \sigma_{\mathrm{N}}}\right)$

$$
(\mathrm{N}=\mathrm{II}, \mathrm{III}, \mathrm{IV}, \mathrm{V})
$$

where

$$
\mu=6,5 m \varepsilon_{0}{ }^{3 / 2} / 2 M
$$

(we take $\mu=1,5 \cdot 10^{-3}$ corresponding to $\varepsilon_{0}=0,9$ ),

$$
\varrho=\frac{n_{1} / n_{\mathrm{e}}}{2 \vartheta^{2} \ln \Lambda}=b_{1} \frac{8 \pi^{3 / 2} e^{\vartheta} v_{+}}{\vartheta^{1 / 2} \ln \left(9 / 32 \pi v_{\mathrm{e}} \vartheta^{3}\right)},
$$

and

$$
\begin{array}{ll}
\sigma_{\mathrm{II}}=f_{12} k_{12} / \varepsilon_{12}{ }^{2}, & \tau_{\mathrm{II}}=f_{12} l_{12} / \varepsilon_{12}, \\
\sigma_{\mathrm{III}}=\sigma_{\mathrm{II}}+f_{13} k_{13} / \varepsilon_{13}{ }^{2}, & \tau_{\mathrm{III}}=\tau_{\mathrm{II}}+f_{13} l_{13} / \varepsilon_{13}
\end{array}
$$

$$
\sigma_{\mathrm{IV}}=\sigma_{\mathrm{III}}+f_{14} k_{14} / \varepsilon_{14}{ }^{2}, \tau_{\mathrm{IV}}=\tau_{\mathrm{III}}+f_{14} l_{14} / \varepsilon_{14}
$$

$$
\sigma_{\mathrm{V}}=\sigma_{\mathrm{IV}}+k_{1} / \varepsilon_{1}^{2}, \quad \tau_{\mathrm{V}}=\tau_{\mathrm{IV}}+l_{1} / \varepsilon_{1} .
$$

\section{Numerical Results and Discussion}

The balance Eqs. (24) for the atomic occupation numbers $b_{i}$, which depend on $\gamma$ through Eqs. (18) and (20), and the Eqs. (42) and (47) for the electron distribuion function $\gamma$, which depend on $b_{i}$ through Eqs. (42) and (50), have been solved numerically by an iterative procedure: Starting with the Maxwell distribution $\gamma^{(0)} \equiv 1$, one calculates the correspon$\operatorname{ding} b_{i}^{(0)}$ and from them the first iterated $\gamma^{(1)}$, and so on. In the average, about 10 iterations were required in order to obtain convergence. In addition to $b_{i}$ and $\gamma$, the quantity $\chi$ [cf. Eq. (9)], which characterizes the degree of ionization, has been calculated; it is given by

$$
\chi=8 \pi^{3 / 2} v_{\mathrm{e}} \vartheta^{3 / 2} \sum_{i=1}^{4} b_{i} i^{2} e^{\vartheta \varepsilon_{i}}
$$

11 M. Abramowitz and I. A. Stegun (Editors), Handbook of Mathematical Functions; Dover, New York 1965. 

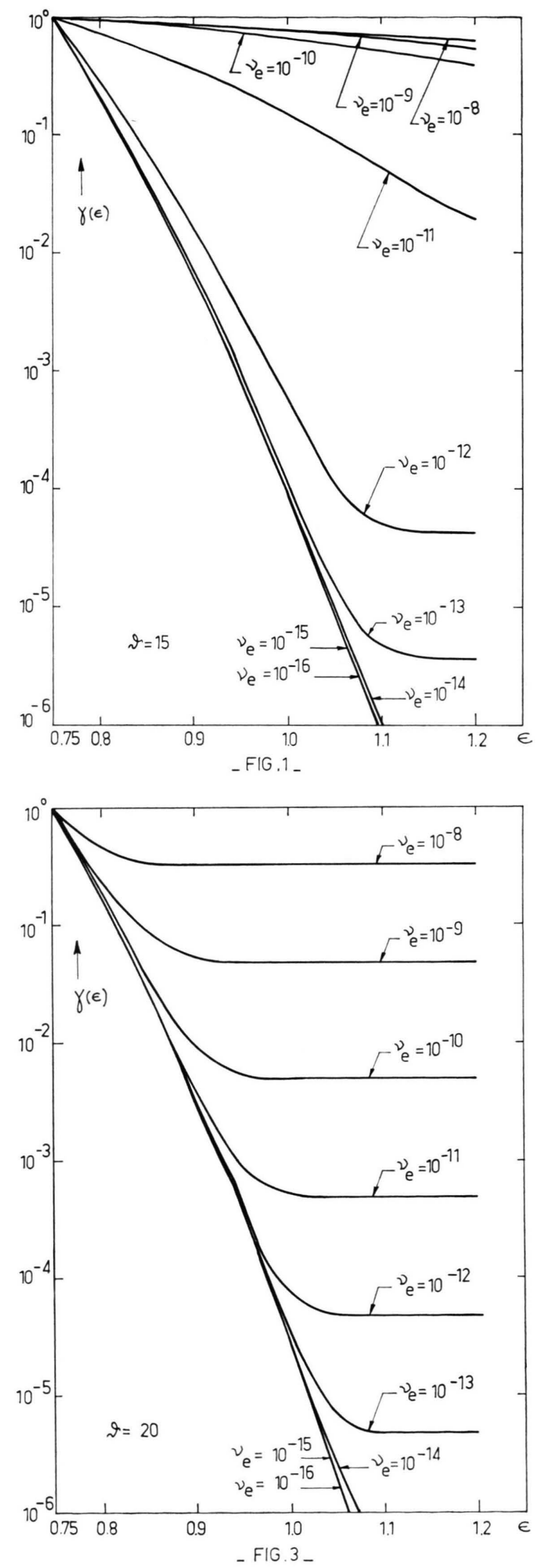

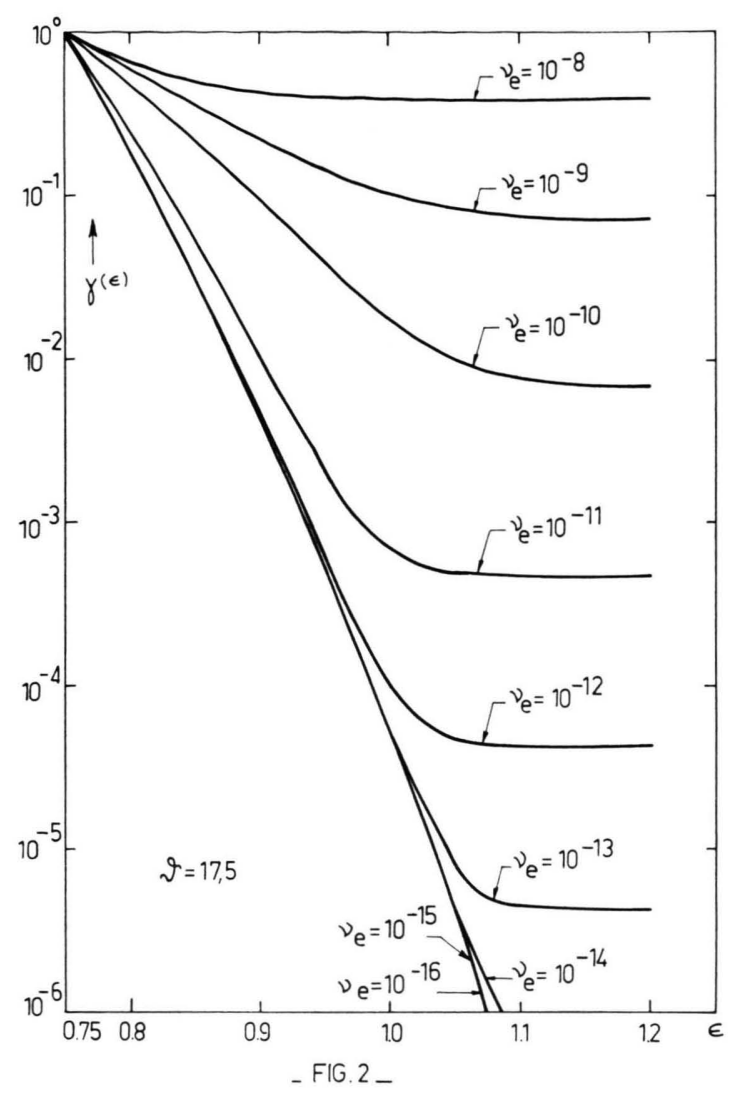

Figs. 1 to 3 . The reduced electron distribution function $\gamma=$ distribution function divided by Maxwell distribution [Eq. (10)] as a function of energy in Rydberg units $\varepsilon=E / I_{\mathrm{H}}$ for different values of temperature $\left(\vartheta=15 \stackrel{=}{T}=10530^{\circ} \mathrm{K}\right.$; $\left.\vartheta=17,5 \triangleq T=9020^{\circ} \mathrm{K} ; \vartheta=20 \triangleq T=7890^{\circ} \mathrm{K}\right)$ and electron density $\left(n_{\mathrm{e}}=6,7 \cdot 10^{24} v_{\mathrm{e}} \mathrm{cm}^{-3}\right)$.

The main results are summarized in Tables 1 to 3 and in Figures 1 to 3.

The at first sight surprisingly strong deviations from the Maxwellian case found for certain temperatures and densities are due to a "feedback effect": On the one hand, the deviations of the distribution function $\gamma$ from a Maxwellian one increase with increasing density ratio $n_{1} / n_{\mathrm{e}}$, and, on the other hand, $n_{1} / n_{\mathrm{e}}$ increases when $\gamma$ becomes smaller since the ionization rate decreases.

It should be noted that the calculated distribution function is likely to overestimate the actual distribution function because 1 . the number of transitions has been underestimated by considering only four bound levels, 2 . the effect of non-resonant transitions on the distribution has been neglected, and 3. the influence of the downward collisions corresponding to resonance transitions has been overestimated. 


\begin{tabular}{|c|c|c|c|c|c|}
\hline & $\begin{array}{c}\vartheta=20 \\
T=7890^{\circ} \mathrm{K}\end{array}$ & $\begin{array}{c}\vartheta=17,5 \\
T=9020^{\circ} \mathrm{K}\end{array}$ & $\begin{array}{c}\vartheta=15 \\
T=10530^{\circ} \mathrm{K}\end{array}$ & $\begin{array}{c}\vartheta=12,5 \\
T \stackrel{12630^{\circ} \mathrm{K}}{ }\end{array}$ & $\begin{array}{c}\vartheta=10 \\
T=15790^{\circ} \mathrm{K}\end{array}$ \\
\hline$\nu_{\mathrm{e}}=10^{-8}$ & 3,$1 ; 0$ & 2,$6 ; 0$ & 1,$9 ; 0$ & 1,$9 ; 0$ & 1,$9 ; 0$ \\
\hline$n_{\mathrm{e}}=6,7 \cdot 10^{16} \mathrm{~cm}^{-3}$ & $(1,7 ; 0)$ & $(1,8 ; 0)$ & $(1,8 ; 0)$ & $(1,9 ; 0)$ & $(1,9 ; 0)$ \\
\hline$v_{\mathrm{e}}=10^{-9}$ & 2,$4 ; 1$ & 1,$6 ; 1$ & 1,$1 ; 1$ & & \\
\hline$n_{\mathrm{e}}==6,7 \cdot 10^{15} \mathrm{~cm}^{-3}$ & $(9,0 ; 0)$ & $(9,3 ; 0)$ & $(9,7 ; 0)$ & & \\
\hline$\nu_{\mathrm{e}}=10^{-10}$ & 5,$0 ; 2$ & 3,$5 ; 2$ & 1,$8 ; 2$ & 1,$6 ; 2$ & 1,$6 ; 2$ \\
\hline$n_{\mathrm{e}}=6,7 \cdot 10^{14} \mathrm{~cm}^{-3}$ & $(1,6 ; 2)$ & $(1,6 ; 2)$ & $(1,6 ; 2)$ & $(1,6 ; 2)$ & $(1,6 ; 2)$ \\
\hline$v_{\mathrm{e}}=10^{-11}$ & 3,$7 ; 4$ & 3,$5 ; 4$ & 1,$6 ; 4$ & & \\
\hline$n_{\mathrm{e}}=6,7 \cdot 10^{13} \mathrm{~cm}^{-3}$ & $(5,0 ; 3)$ & $(4,8 ; 3)$ & $(4,5 ; 3)$ & & \\
\hline$v_{\mathrm{e}}=10^{-12}$ & 4,$4 ; 6$ & 4,$9 ; 6$ & 4,$8 ; 6$ & 4,$1 ; 4$ & 3,$7 ; 4$ \\
\hline$n_{\mathrm{e}}=6,7 \cdot 10^{12} \mathrm{~cm}^{-3}$ & $(3,7 ; 4)$ & $(3,7 ; 4)$ & $(3,7 ; 4)$ & $(3,7 ; 4)$ & $(3,6 ; 4)$ \\
\hline$v_{\mathrm{e}}=10^{-13}$ & 4,$2 ; 8$ & 4,$9 ; 8$ & 5,$6 ; 8$ & & \\
\hline$n_{\mathrm{e}}=6,7 \cdot 10^{11} \mathrm{~cm}^{-3}$ & $(2,9 ; 5)$ & $(3,0 ; 5)$ & $(3,0 ; 5)$ & & \\
\hline$v_{\mathrm{e}}=10^{-14}$ & 4,$1 ; 10$ & 4,$6 ; 10$ & 5,$2 ; 10$ & 3,$3 ; 6$ & 3,$2 ; 6$ \\
\hline$n_{\mathrm{e}}=6,7 \cdot 10^{10} \mathrm{~cm}^{-3}$ & $(2,8 ; 6)$ & $(2,9 ; 6)$ & $(3,0 ; 6)$ & $(3,0 ; 6)$ & $(3,1 ; 6)$ \\
\hline$v_{\mathrm{e}}=10^{-15}$ & 3,$3 ; 12$ & 3,$3 ; 12$ & 3,$1 ; 12$ & & \\
\hline$n_{\mathrm{e}}=6,7 \cdot 10^{9} \mathrm{~cm}^{-3}$ & $(2,8 ; 7)$ & $(2,9 ; 7)$ & $(3,0 ; 7)$ & & \\
\hline$v_{\mathrm{e}}=10^{-16}$ & 1,$1 ; 14$ & 8,$5 ; 13$ & 6,$1 ; 13$ & 3,$2 ; 8$ & 3,$1 ; 8$ \\
\hline$n_{\mathrm{e}}=6,7 \cdot 10^{8} \mathrm{~cm}^{-3}$ & $(2,8 ; 8)$ & $(2,9 ; 8)$ & $(3,0 ; 8)$ & $(3,0 ; 8)$ & $(3,1 ; 8)$ \\
\hline
\end{tabular}

Table 1. The quantity $b_{1}=n_{1} / n_{1}$ Saha [Eq. (8)]. Without brackets: self-consistently calculated values. With brackets: values corresponding to a Maxwell distribution of the electrons. Read: 3,$7 ; 4=3,7 \cdot 10^{4}$.

\begin{tabular}{|c|c|c|c|c|c|}
\hline & $\begin{array}{c}\vartheta=20 \\
T=7890^{\circ} \mathrm{K}\end{array}$ & $\begin{array}{c}\vartheta=17,5 \\
T=9020^{\circ} \mathrm{K}\end{array}$ & $\begin{array}{c}\vartheta=15 \\
T=10530^{\circ} \mathrm{K}\end{array}$ & $\begin{array}{c}\vartheta=12,5 \\
T=12630^{\circ} \mathrm{K}\end{array}$ & $\begin{array}{c}\vartheta=10 \\
T=15790^{\circ} \mathrm{K}\end{array}$ \\
\hline$v_{\mathrm{e}}=10^{-8}$ & 1,$0 ; 0$ & 1,$0 ; 0$ & 1,$0 ; 0$ & 1,$0 ; 0$ & 1,$0 ; 0$ \\
\hline$n_{\mathrm{e}}=6,7 \cdot 10^{16} \mathrm{~cm}^{-3}$ & $(1,0 ; 0)$ & $(1,0 ; 0)$ & $(1,0 ; 0)$ & $(1,0 ; 0)$ & $(1,0 ; 0)$ \\
\hline$v_{\mathrm{e}}=10^{-9}$ & 1,$1 ; 0$ & 1,$1 ; 0$ & 1,$1 ; 0$ & & \\
\hline$n_{\mathrm{e}}=6,7 \cdot 10^{15} \mathrm{~cm}^{-3}$ & $(1,1 ; 0)$ & $(1,1 ; 0)$ & $(1,1 ; 0)$ & & \\
\hline$v_{\mathrm{e}}=10^{-10}$ & 2,$4 ; 0$ & 2,$3 ; 0$ & 2,$1 ; 0$ & 2,$0 ; 0$ & 1,$9 ; 0$ \\
\hline$n_{\mathrm{e}}=6,7 \cdot 10^{14} \mathrm{~cm}^{-3}$ & $(2,3 ; 0)$ & $(2,2 ; 0)$ & $(2,1 ; 0)$ & $(2,0 ; 0)$ & $(1,9 ; 0)$ \\
\hline$v_{\mathrm{e}}=10^{-11}$ & 1,$7 ; 1$ & 1,$7 ; 1$ & 1,$4 ; 1$ & & \\
\hline$n_{\mathrm{e}}=6,7 \cdot 10^{13} \mathrm{~cm}^{-3}$ & $(7,3 ; 0)$ & $(6,6 ; 0)$ & $(6,0 ; 0)$ & & \\
\hline$v_{\mathrm{e}}=10^{-12}$ & 2,$1 ; 2$ & 2,$1 ; 2$ & 2,$1 ; 2$ & 5,$1 ; 0$ & 4,$5 ; 0$ \\
\hline$n_{\mathrm{e}}=6,7 \cdot 10^{12} \mathrm{~cm}^{-3}$ & $(5,3 ; 0)$ & $(5,1 ; 0)$ & $(4,9 ; 0)$ & $(4,7 ; 0)$ & $(4,4 ; 0)$ \\
\hline$v_{\mathrm{e}}=10^{-13}$ & 2,$0 ; 3$ & 2,$0 ; 3$ & 2,$1 ; 3$ & & \\
\hline$n_{\mathrm{e}}=6,7 \cdot 10^{11} \mathrm{~cm}^{-3}$ & $(4,2 ; 0)$ & $(4,2 ; 0)$ & $(4,1 ; 0)$ & & \\
\hline$v_{\mathrm{e}}=10^{-14}$ & 1,$9 ; 4$ & 1,$9 ; 4$ & 1,$9 ; 4$ & 4,$1 ; 0$ & 3,$8 ; 0$ \\
\hline$n_{\mathrm{e}}=6,7 \cdot 10^{10} \mathrm{~cm}^{-3}$ & $(4,1 ; 0)$ & $(4,0 ; 0)$ & $(4,0 ; 0)$ & $(3,9 ; 0)$ & $(3,8 ; 0)$ \\
\hline$v_{\mathrm{e}}=10^{-15}$ & 1,$5 ; 5$ & 1,$4 ; 5$ & 1,$1 ; 5$ & & \\
\hline$n_{\mathrm{e}}=6,7 \cdot 10^{9} \mathrm{~cm}^{-3}$ & $(4,1 ; 0)$ & $(4,0 ; 0)$ & $(4,0 ; 0)$ & & \\
\hline$v_{\mathrm{e}}=10^{-16}$ & 5,$2 ; 5$ & 3,$6 ; 5$ & 2,$3 ; 5$ & 4,$1 ; 0$ & 3,$8 ; 0$ \\
\hline$n_{\mathrm{e}}=6,7 \cdot 10^{8} \mathrm{~cm}^{-3}$ & $(4,1 ; 0)$ & $(4,0 ; 0)$ & $(4,0 ; 0)$ & $(3,9 ; 0)$ & $(3,8 ; 0)$ \\
\hline
\end{tabular}

Table 2. The quantity $b_{2}=n_{2} / n_{2}$ Saha [Eq. (8)]. Without brackets: self-consistently calculated values. With brackets: values corresponding to a Maxwell distribution of the electrons. Read: 3,$7 ; 4=3,7 \cdot 10^{4}$.

\begin{tabular}{|c|c|c|c|c|c|}
\hline & $\begin{array}{c}\vartheta=20 \\
T=7890^{\circ} \mathrm{K}\end{array}$ & $\begin{array}{c}\vartheta=17,5 \\
T=9020^{\circ} \mathrm{K}\end{array}$ & $\begin{array}{c}\vartheta=15 \\
T=10530^{\circ} \mathrm{K}\end{array}$ & $\begin{array}{c}\vartheta=12,5 \\
T \stackrel{12630^{\circ} \mathrm{K}}{ }\end{array}$ & $\begin{array}{c}\vartheta=10 \\
T=15790^{\circ} \mathrm{K}\end{array}$ \\
\hline $\begin{array}{l}v_{\mathrm{e}}=10^{-8} \\
n_{\mathrm{e}}=6,7 \cdot 10^{16} \mathrm{~cm}^{-3} \\
v_{\mathrm{e}}=10^{-9} \\
n_{\mathrm{e}}=6,7 \cdot 10^{15} \mathrm{~cm}^{-3} \\
v_{\mathrm{e}}=10^{-10} \\
n_{\mathrm{e}}=6,7 \cdot 10^{14} \mathrm{~cm}^{-3} \\
v_{\mathrm{e}}=10^{-11} \\
n_{\mathrm{e}}=6,7 \cdot 10^{13} \mathrm{~cm}^{-3} \\
v_{\mathrm{e}}=10^{-12} \\
n_{\mathrm{e}}=6,7 \cdot 10^{12} \mathrm{~cm}^{-3} \\
v_{\mathrm{e}}=10^{-13} \\
n_{\mathrm{e}}=6,7 \cdot 10^{11} \mathrm{~cm}^{-3} \\
v_{\mathrm{e}}=10^{-14} \\
n_{\mathrm{e}}=6,7 \cdot 10^{10} \mathrm{~cm}^{-3} \\
v_{\mathrm{e}}=10^{-15} \\
n_{\mathrm{e}}=6,7 \cdot 10^{9} \mathrm{~cm}^{-3} \\
v_{\mathrm{e}}=10^{-16} \\
n_{\mathrm{e}}=6,7 \cdot 10^{8} \mathrm{~cm}^{-3}\end{array}$ & $\begin{array}{c}6,0 ; 4 \\
(3,3 ; 4) \\
4,6 ; 4 \\
(1,7 ; 4) \\
9,6 ; 4 \\
(3,1 ; 4) \\
7,1 ; 5 \\
(9,6 ; 4) \\
8,6 ; 6 \\
(7,1 ; 4) \\
8,2 ; 7 \\
(5,6 ; 4) \\
7,9 ; 8 \\
(5,4 ; 4) \\
6,3 ; 9 \\
(5,4 ; 4) \\
2,1 ; 10 \\
(5,4 ; 4)\end{array}$ & $\begin{array}{c}3,4 ; 3 \\
(2,3 ; 3) \\
2,0 ; 3 \\
(1,2 ; 3) \\
4,6 ; 3 \\
(2,1 ; 3) \\
4,6 ; 4 \\
(6,2 ; 3) \\
6,4 ; 5 \\
(4,8 ; 3) \\
6,3 ; 6 \\
(3,9 ; 3) \\
6,0 ; 7 \\
(3,7 ; 3) \\
4,3 ; 8 \\
(3,7 ; 3) \\
1,1 ; 9 \\
(3,7 ; 3)\end{array}$ & $\begin{array}{c}1,6 ; 2 \\
(1,5 ; 2) \\
8,9 ; 1 \\
(8,2 ; 1) \\
1,6 ; 2 \\
(1,3 ; 2) \\
1,3 ; 3 \\
(3,8 ; 2) \\
4,1 ; 4 \\
(3,1 ; 2) \\
4,7 ; 5 \\
(2,6 ; 2) \\
4,4 ; 6 \\
(2,5 ; 2) \\
2,6 ; 7 \\
(2,5 ; 2) \\
5,2 ; 7 \\
(2,5 ; 2)\end{array}$ & $\begin{array}{c}2,1 ; 1 \\
(1,9 ; 1)\end{array}$ & $\begin{array}{c}5,0 ;-1 \\
(5,0 ;-1) \\
1,1 ; 0 \\
(1,1 ; 0)\end{array}$ \\
\hline
\end{tabular}

Table 3. The quantity $\chi=n_{\mathrm{H}} / n_{+}$[Eq. (9)]. Without brackets: self-consistently calculated values. With brackets: values corresponding to a Maxwell distribution of the electrons. Read: 3,$7 ; 4=3,7 \cdot 10^{4}$. 
The Maxwellian values in Tables 1 to 3 are in fair agreement with previously published ones ${ }^{4,5}$; differences, up to factors of about 3 , are essentially due to the use of a cross section $Q_{12}$ that is finite at threshold.

The results indicate that below $10000{ }^{\circ} \mathrm{K}$ the degree of ionization is smaller than it had been as- sumed up to now. Accordingly, the effects of radiative transfer and of inelastic atom-atom collisions will be more important there.

I wish to thank Miss N. Benoit for having written the computer program.

\title{
Messungen der radialen Verteilung der elektrischen Feldstärke im Wasserstofflichtbogen mit axialem Magnetfeld*
}

\author{
R. SCHWENN \\ Institut für Plasmaphysik, Garching bei München \\ (Z. Naturforsch. 25 a, 1310-1316 [1970] ; eingegangen am 3. Juni 1970)
}

\begin{abstract}
In a stationary hydrogen arc with axial magnetic field the radial distribution of the axial electric field $E_{z}$ was measured by shooting a double potential probe across the arc. It was found that $E_{z}$ strongly decreases outside the magnetic flux tube defined by the cathode radius and tends to zero. The measured distribution of $E_{z}$ agrees qualitatively with computations allowing for the observed rotation profile. In a very fast rotating arc with a hollow anode $E_{z}$ was found to vanish not only outwards, but also towards the axis, as predicted by the theory.
\end{abstract}

\section{Einführung}

Ein starkes Magnetfeld, das einem Lichtbogen axial überlagert wird, reduziert die radialen Wärmeverluste $^{1-3}$. Deshalb konnten in stationären Wasserstofflichtbögen (Bogenstrom $\approx 2000 \mathrm{~A}$, Magnetfeld $10-40 \mathrm{kG}$, Druck $1-10$ Torr, Länge $10-40 \mathrm{~cm}$ ) schon Temperaturen bis $150000^{\circ} \mathrm{K}$ erzielt werden ${ }^{4,5}$. Bei diesen Experimenten war der Radius des Bogens stets sehr viel kleiner als der des ihn umgebenden Gefäßes und auch unabhängig davon. Die bekannte Theorie für wandstabilisierte zylindersymmetrische (d.h. unendlich lange) Bögen kann in diesem Fall offensichtlich nicht angewendet werden. Gibt man allerdings als Gefäßradius den optisch bestimmten „Bogenradius“ vor, dann erhält man nach dieser Theorie Temperaturprofile, die mit den gemessenen gut übereinstimmen ${ }^{4,6}$. Die Frage, wodurch dieser Bogenradius bestimmt wird, blieb offen.

\footnotetext{
* Auszug aus der von der Fakultät für Maschinenwesen und Elektrotechnik der Technischen Hochschule München genehmigten Dissertation über „Sondenmessungen der radialen Verteilungen von Potential, elektrischer Feldstärke und Stromdichte im Wasserstofflichtbogen mit starkem überlagertem Magnetfeld“.

Sonderdruckanforderungen an R. ScHWENN, D-8046 Garching, Münchner Str. 22.

1 R. WIENECKe, Z. Naturforsch. 19 a, 675 [1964].
}

RAEDER und Wirtz ${ }^{7}$ haben deshalb für einen Bogen endlicher Länge und mit Elektroden endlichen Durchmessers ohne Annahmen über einen „Bogenradius" die Potentialgleichung und die Energiegleichung mit numerischen Verfahren simultan gelöst. Daraus ergaben sich die Verteilungen von Potential, elektrischem Feld, Stromdichte und Temperatur in ganzen Bogen. Ein wichtiges Ergebnis war, daß die axiale elektrische Feldstärke $E_{z}$ außerhalb der durch die Elektroden bestimmten magnetischen Flußröhre abnehmen und gegen Null gehen sollte (im Gegensatz dazu fordert die Annahme von Zylindersymmetrie, daß $E_{z}$ über den Radius konstant ist). Eine Rotation des Plasmas sollte diese Tendenz verstärken. Dies lassen auch KLÜBERs Messungen ${ }^{8}$ an einem Heliumbogen („Eieruhr“) erkennen.

Über den detaillierten radialen Verlauf von $E_{z}$ ist bisher jedoch noch nichts bekannt. Zur Klärung dieser Zusammenhänge sollten die im folgenden beschriebenen Experimente beitragen.

2 C. Mahn, H. Ringler, R. Wienecke, S. Witkowski u. G. ZANKL, Z. Naturforsch. 19 a, 1202 [1964].

3 J. Raeder u. S. Wirtz, Z. Naturforsch. 23 a, 1695 [1968].

4 C. Mahn, H. Ringler u. G. ZankL, Z. Naturforsch. 23 a, 867, 874 [1968] ; Phys. Lett. 27 A, 683 [1968].

5 D. Ludwig, Inst. für Plasmaphysik, Garching, Rep. 3/88 [1969]; Z. Naturforsch. (wird veröffentlicht).

6 U. HeIdrich, Z. Naturforsch. 20 a, 475 [1965].

7 J. Raeder u. S. Wirtz, Z. Naturforsch. 25 a, 459 [1970].

8 O. Klüber, Z. Naturforsch. 24 a, 1473 [1969]. 